\title{
Moderne ärztlich-kollegiale Kommunikation im Gesundheitswesen
}

\section{Bernhard Schaller ${ }^{a}$, Gaby Baller ${ }^{b}$}

a Universitätsklinikum Münster/D

b GMHM mbH, Berlin, Mannheim, Heidelberg; Institut für Medizincontrolling, Heidelberg/D

Korrespondenz:

Gaby Baller

GMHM mbH

Rohrbacher Strasse 92/1

D-69115 Heidelberg

www.gmhm.de

www.drg24.ch
Für die bestehenden und zukünftigen Aufgaben in dem sich im Wandel befindenden Gesundheitswesen sind zunehmend Schlüsselkompetenzen gefragt [1], die die geforderte Patientenorientierung unterstützen. Dies nicht zuletzt vor dem Hintergrund, da im Gesundheitswesen der Zukunft immer mehr der Patient als Kunde im Vordergrund stehen wird, was heutzutage eine Verwaltung voraussetzt, die sich als Dienstleisterin versteht und ihre Beschäftigten in diesem Paradigmenwechsel durch entsprechende Fortbildungsmassnahmen zur Entwicklung sozialer Kompetenzen und Serviceorientierung unterstützt. Ebenso sind bei der Personalauswahl Zuwendung zu Patienten und Angehörigen sowie Teamfähigkeit als Voraussetzung zu beachten, um eine Ärzteschaft zu entwickeln, die den heutigen Anforderungen entspricht. Lange Zeit hat es genügt, allein fachliche Kompetenz $\mathrm{zu}$ besitzen. Das ist in der heutigen Zeit nicht mehr möglich. Denn es kommt vielmehr darauf an, ein reibungslos funktionierendes Team zu haben, das effizient arbeitet und seine Zeit nicht mit Kleinkriegen verbringt, wie es die Autoren schon sehr häufig erlebt haben. Allein hier ist Einsparpotential, wie es die Privatwirtschaft schon längst erkannt hat, die ihre Mitarbeiter entsprechend auswählt und regelmässig, nicht nur fachlich, sondern eben auch in den sogenannten weichen Faktoren schult.

Neben der externen kollegialen Kommunikation ist daher auch die interne kollegiale Kommunikation zunehmend bedeutsam [1], gerade in den aktuellen Veränderungsprozessen des Gesundheitswesens ist sie zur Optimierung der Arbeitsprozesse unabdingbar. Ein funktionierendes Team ist vor allem auch auf partnerschaftliches Verhalten mit relativ intensiven wechselseitigen Beziehungen zurückzuführen. Auf diese (neuen) Herausforderungen wird in der Ausbildung bisher nur unzureichend bis gar nicht vorbereitet, interessanterweise ebensowenig wie betriebswirtschaftliche Kenntnisse, die heutzutage von Ärzten verlangt werden. Wir wollen im Folgenden - nach unseren theoretischen Ausführungen [1] - auch anhand zweier konkreter Beispiele die Wichtigkeit einer ärztlich-kollegialen Kommunikation im Gesundheitswesen herausstreichen.
Im soziotechnischen System «Operationssaal» kooperieren Fachleute unterschiedlicher Disziplinen. Jede Operation verlangt daher Teamarbeit. Neben der Koordination des gesamten Operationsteams während einer «normalen» Operation ist vor allem bei Not- und Zwischenfällen kooperatives Handeln gefragt. An solch risikobelasteten Arbeitsplätzen können Missverständnisse fatale Folgen haben. Probleme entstehen vor allem durch Zeitdruck und eingeschränkte Problemlösungsressourcen. Dadurch wird Kooperation erschwert, und deshalb kommt der Kommunikation im Operationssaal eine enorme Bedeutung zu: mangelnder Teamgeist, sprachliche Unzulänglichkeiten, stressbedingter Informationsstau sowie die Unfähigkeit, angemessen kritisches Feedback geben zu können. Solcherart sind die Kommunikationsprobleme im Operationssaal. Da die Teamkommunikation im Operationssaal ein relativ junges Forschungsgebiet ist, können der vergleichsweise besser erforschte und dokumentierte Hochrisikoarbeitsplatz Luftfahrt und die Cockpitkommunikation vielleicht erste Hinweise liefern: Hier werden bis zu 70\% der Zwischenfälle auf schlechte, d.h. oft zuwenig Teamkommunikation zurückgeführt. Daraus folgt, dass für die Effektivität und damit den Erfolg eines Teams in einer ganz spezifischen Weise kommuniziert werden muss: Knappe, einfache, aber zugleich präzise und unmissverständliche Formulierungen sind geeignet, limitierte kognitive Ressourcen für die Lösung der primären Aufgabe freizuhalten und damit zur Vermeidung von «Bottleneck»-Effekten, auch als Flaschenhalseffekt bezeichnet. Damit ist die Schwierigkeit innerhalb menschlicher Kommunikation gemeint, die kognitive Aufnahmekapazität gleichzeitig auf mehrere zentrale Prozesse zu richten bzw. unter solchen Doppelaufgabenbedingungen gleichzeitig die Teamkooperation und -koordination zu fördern. Dafür greifen gutorganisierte Teams auf grammatische, interaktive und kommunikative Kompetenz zurück. Eine solche «Teamsprache» ist auch geeignet, das in kritischen Situationen geforderte Wir-Gefühl des Teams zu fördern, was gerade im Operationssaal eine wichtige und daher anzustrebende Teamentwicklung ist, die regelmässig trainiert werden muss. Dessen Etablierung als Routine 
spricht für eine hohe kommunikative Kompetenz. Zusätzlich sehen - nach unseren Beobachtungen aus Angst vor Sanktionen - Ärzte und Pflegepersonal starke Hierarchien als Hemmnis, Fehler anzusprechen.

Deshalb sind schnittstellenübergreifende Teamentwicklungen und Trainings nicht nur sinnvoll, sondern wohl unabdingbar, um die Arbeitseffizienz des Teams zu steigern. Oft werden dabei nicht nur Kompetenzen einzelner Teammitglieder oder der ganzen Gruppe (z.B. Kommunikation) optimiert, sondern auch Strukturen der Zusammenarbeit neu geordnet. Es gab in Deutschland im Jahre 1997 den Fall eines 7jährigen Mädchens, das ein leichtes Schädel-Hirn-Trauma erlitten hatte. Im Rahmen der primären notärztlichen Versorgung auf dem Unfallplatz hatte die sehr gutausgebildete Notärztin versucht, eine Narkose einzuleiten, wobei mehrere Intubationsversuche fehlschlugen und das Kind schliesslich an einer Universitätsklinik in Deutschland angeblich an Hypoxie verstarb [2]. Eine Autopsie hat nie stattgefunden. Ein tragisches Schicksal, wäre da nicht die Auskunft durch den letztbehandelnden Arzt an die Eltern, dass das Kind durch eine Fehlintubation, also durch einen Kunstfehler, gestorben sei, den die zuerst behandelnde Notärztin zu verantworten habe. Hier hat der letztbehandelnde Arzt aufgrund einer eigenen, objektiv kaum nachvollziehbaren Hypothese eine kollegiale Abrechnung in Gang gesetzt, die schliesslich nach wissenschaftlichen Kriterien nie beweisbar war. Was mochte er damit bewirken? [3]. Er möchte sich selbst erheben und sagen, dass ihm das nie passiert wäre? An die Ärzte appellieren, dass qualitativ besser gearbeitet werden soll? Den Eltern das Gefühl geben, dass allein Ärzte über Leben und Tod durch Können oder Nichtkönnen entscheiden? Hier fehlte ganz offenbar der Nährboden, auf dem erfolgreiches Arbeiten gedeihen kann: Die Kultur der Kommunikation und Zusammenarbeit, ob in Krisen oder Hochleistungsperioden, ist grundsätzlich notwendig zur Organisationsentwicklung. Nach heutiger Interpretation des Risikomanagements ist hier aber nicht die behandelnde Notärztin schuld, sondern es ist das System an sich, das solche Fehler zulässt. Es lässt sich aber nicht voraussagen, welchen Notarzt und welches Kind dieses Schicksal tref- fen wird. Vielmehr ist es meist eine Kette unvorhersehbarer kritischer Ereignisse, die ein solches Desaster verursachen. Ärzte, die sich selbst für unfehlbar halten, können ihren Kollegen schwerlich den Wert einer konstruktiven Fehleranalyse vermitteln. Viele Rechtsstreitigkeiten entstehen letztendlich nicht, weil ein Patient geschädigt wurde, sondern weil er nach seiner Schädigung in verletzender und respektloser Weise von Ärzten behandelt wurde.

Von der Art und Weise, wie im Unternehmen oder unter ärztlichen Kollegen zusammengearbeitet wird, hängt $\mathrm{ab}$, mit wieviel oder wie wenig Reibungsverlusten Prozesse funktionieren. Wahrscheinlich haben wir alle schon die Erfahrung gemacht, wie schwer es ist, festgefahrene Muster aufzubrechen und eingespielte Konfliktlinien aufzulösen, um wieder produktiv zusammenarbeiten zu können. Aber konstruktive Fehleranalysen und daraus zu lernen sind Erfolgsfaktoren, nicht nur im Krankenhaus.

Das Vertrauen der Patienten ist ein wichtiger Erfolgsfaktor im heutigen Gesundheitswesen. Dieses Vertrauen orientiert sich stark an der gesellschaftlichen Risikoakzeptanz, die wir Ärzte durch unser Kommunikationsverhalten steuern können. Emotionale Intelligenz, Fachkompetenz und eine Handlungsintelligenz, in den entscheidenden Situationen die richtigen Dinge zu tun, sollten vorhanden sein. Ebenso Transparenz über Handlungsweisen und die Hintergründe, damit es keinen Raum für Interpretationen gibt. Und dort, wo es noch keine ausreichenden Informationen gibt, darf man auch keine Spekulationen anstellen. Wer möchte, dass er/sie oder seine/ihre Arbeit respektiert und wertgeschätzt wird, muss die Arbeit der anderen respektieren. Und: Wer die Würde der anderen wahrt, wahrt auch die eigene. Patienten gehen dahin, wo es ihnen rundum gefällt, und deshalb ist schon lange nicht mehr nur gute Medizin ausschlaggebend für den Erfolg eines Krankenhauses.

\section{Literatur}

1 Schaller B, Baller G. In varietate concordia oder Abrechnungsmentalität unter Kollegen. Schweiz Ärztezeitung. 2007;88(39):1641-3.

2 www.stefanie-bachstein.de.

3 Schulz von Thun F. Miteinander reden. Reinbek: Rowohlt; 1981. 